\title{
Expression of NAMPT is associated with breast invasive ductal carcinoma development and prognosis
}

\author{
SHAO-JIE ZHOU ${ }^{1}$, TIE-QIANG BI ${ }^{1}$, CHUN-XIN QIN $^{1}$, XIAO-QING YANG $^{1}$ and KAI PANG ${ }^{2}$ \\ Departments of ${ }^{1}$ Thyroid and Breast Surgery and ${ }^{2}$ Statistics, Weihai Municipal Hospital, \\ Weihai, Shandong 264200, P.R. China
}

Received May 3, 2017; Accepted January 19, 2018

DOI: $10.3892 / \mathrm{ol} .2018 .8164$

\begin{abstract}
Nicotinamide phosphoribosyltransferase (NAMPT) possesses various functions in human cells, and altered NAMPT expression is associated with human carcinogenesis. The present study detected the expression of NAMPT in normal and cancerous breast tissues from 83 patients using immunohistochemistry, and analyzed its association with the clinicopathological and survival data of the patients. NAMPT was significantly overexpressed in the breast invasive ductal carcinoma tissues compared with adjacent normal mammary gland tissues. Upregulated NAMPT expression was associated with a larger tumor size, lymph node metastasis, advanced clinical tumor-node-metastasis stages, and estrogen receptor and progesterone receptor expression. Furthermore, NAMPT expression was associated with poor overall and disease-free survival in patients with breast cancer. In conclusion, NAMPT increased protein expression in tumor cells may contribute to the development and progression of breast invasive ductal carcinoma. Thus, detection of NAMPT expression might be useful as a biomarker for the early detection and prognosis prediction of breast cancer.
\end{abstract}

\section{Introduction}

Breast cancer is a significant health problem in women globally, and breast cancer incidence rates are higher in North America and Western Europe, compared with those in other parts of the world. In the United States in 2016, there were 249,260 new cases of breast cancer and 40,890 cancer-related deaths (1). In China, breast cancer has become one of the most frequently diagnosed cancers in women aged 30-59 years old (2), and a high proportion of the patients are diagnosed at the advanced stages of disease (2); for those patients, treatment

Correspondence to: Dr Tie-Qiang Bi, Department of Thyroid and Breast Surgery, Weihai Municipal Hospital, 70 Heping Road, Weihai, Shandong 264200, P.R. China

E-mail: 37400704@qq.com

Key words: breast cancer, nicotinamide phosphoribosyltransferase, immunohistochemistry, prognosis responses, even with post-surgical chemotherapy and radiotherapy, remain poor (2). Thus, novel molecular biomarkers are required for the early detection and treatment response prediction in patients with breast cancer.

Mammalian cell nicotinamide phosphoribosyltransferase (NAMPT) is the rate-limiting enzyme in the biosynthesis of nicotinamide adenine dinucleotide (NAD) that is responsible for transferring a phosphoribosyl group from 5-phosphoribosyl-1-pyrophosphate to nicotinamide, resulting in the production of nicotinamide mononucleotide (NMN) and pyrophosphate. NMN is then converted to NAD by NMN adenylyltransferase (Fig. 1) (3). Increasing evidence suggests that NAMPT is a multifunctional enzyme with crucial roles in in metabolism and immune response, and altered NAMPT expression is associated with human tumorigenesis (4). Silencing of NAMPT expression in pancreatic cancer cells has been demonstrated to induce tumor cell metabolic collapse and cell death both in vitro and in vivo (5), whereas NAMPT overexpression is associated with poor treatment response of breast cancer patients to doxorubicin-based chemotherapy (6). Previous studies from our group have demonstrated that NAMPT is highly expressed in gastric cancer and is associated with malignant behaviors of cancer cells as well as resistance to chemotherapy $(7,8)$. The aim of the present study was to detect NAMPT expression in normal and cancerous breast tissues using immunohistochemistry and to examine its association with clinicopathological and survival data from breast cancer patients.

\section{Materials and methods}

Patient samples. Paired cancerous and adjacent noncancerous breast tissues were collected from 83 newly diagnosed and surgically treated breast cancer patients at Weihai Municipal Hospital (Weihai, China) between January and December 2008. The adjacent normal tissues were $>5 \mathrm{~cm}$ away from the tumor lesions. All patients were histologically diagnosed with invasive ductal carcinoma and aged 29-66 years old, with an average age of 47 years. None of the patients received presurgical radiotherapy or chemotherapy. The present study was approved by the Ethics Committee of Weihai Municipal Hospital, and informed consent was obtained from each patient. The histologic types and grades of the primary tumors were determined according to the modifications of 


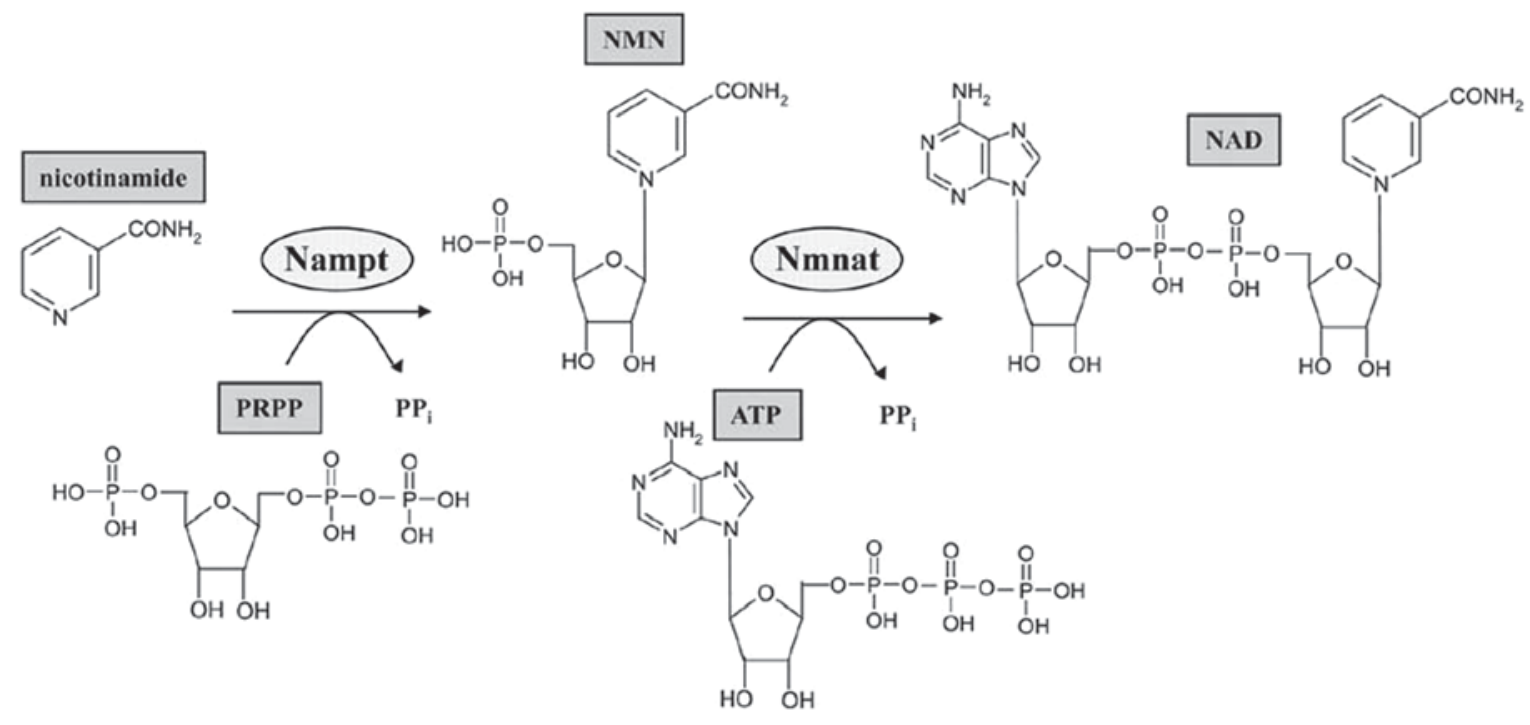

Figure 1. The NAD biosynthesis pathway. NAMPT can transfer a phosphoribosyl group obtained from PRPP to nicotinamide, resulting in the production of NMN and PPi. Thereafter, NMN is converted to NAD by NMNAT. NAD, nicotinamide adenine dinucleotide; NAMPT, nicotinamide phosphoribosyltransferase; PRPP, 5-phosphoribosyl-1-pyrophosphate; NMN, nicotinamide mononucleotide; PPi, pyrophosphate; NMNAT, nicotinamide mononucleotide adenylyltransferase.

the World Health Organization classification (9), whereas the staging of breast cancer was defined according to the tumor-node-metastasis (TNM) system (10).

Immunohistochemistry. All tissue samples were fixed in $10 \%$ buffered formalin at room temperature for $24 \mathrm{~h}$ and subsequently embedded into paraffin. Tissue sections $4-\mu \mathrm{m}$ thick were then prepared from these paraffin blocks and immunohistochemically stained using the streptavidin peroxidase (SP) technique. In brief, tissue sections were deparaffinized in xylene, rehydrated in a series of ethanol solutions, and then submerged in tap water. The tissue sections were subjected to antigen retrieval in a pressure cooker containing $0.01 \mathrm{M}$ citrate buffer and blocking of the peroxidase activity in $3 \% \mathrm{H}_{2} \mathrm{O}_{2}$ for $30 \mathrm{~min}$ at room temperature. Next, they were incubated with $20 \%$ normal goat serum (Abcam, Cambridge, MA, USA) diluted in PBS for $30 \mathrm{~min}$ and then with an anti-NAMPT antibody (cat. no., ab45890; 1:50; Abcam) at $4^{\circ} \mathrm{C}$ overnight. The next day, the tissues sections were washed with PBS briefly three times, and then incubated with a goat anti-mouse immunoglobulin G (cat. no., sc-2039; 1:200; Santa Cruz Biotechnology, Inc., Dallas, TX, USA) conjugated with SP for $30 \mathrm{~min}$ at room temperature. After washing with PBS, the tissue sections were subjected to a colorimetric reaction using 3,3'-diaminobenzidine solution, then counterstained with hematoxylin briefly, mounted with mounting medium, and covered with a coverslip. The immunostained tissue sections were reviewed and photographed under a light microscope. Image acquisition and analysis were then performed. Positively stained cells appeared brown or displayed brown cytoplasmic granules in the cytoplasm. NAMPT immunostaining scores were based on the intensity of the immunostaining and the $\%$ of positively stained cells. Immunostaining intensity was scored as follows: 0 , no staining; 1 , weak staining; 2 , moderate staining; and 3, strong staining. Percentage of positive staining was scored as follows; $1, \leq 25 \%$ positive cells; $2,26-50 \%$ positive cells; $3,51-75 \%$ positive cells; and $4, \geq 76 \%$ positive cells. The sum of these two scores resulted in a final score for each case to determine high vs. low expression of NAMPT protein (a score of $\geq 3$ was termed high NAMPT expression, whereas a score of 1-2 was termed low NAMPT expression). The staining of each tissue section was scored separately by two independent experts simultaneously, and discordant scores were re-evaluated and scored with their consensual opinion.

Statistical analysis. All statistical analyses were performed using the SPSS version 17.0 software (SPSS, Inc., Chicago, IL, USA). Comparisons of NAMPT expression with clinicopathological parameters, including tumor stage, tumor grade, age at diagnosis, body mass index, tumor size, lymph node status, recurrence, estrogen receptor (ER) status, progesterone receptor (PR) status, and human epidermal growth factor receptor 2 (HER2) status, were analyzed using the $\chi^{2}$ or Fisher's exact test (when a cell in a $2 \times 2$ table had an expected frequency of $\leq 5$ ). Survival curves were generated using Kaplan-Meier curves and statistically analyzed using the log-rank test. Multivariate analysis was performed using Cox's proportional hazard model. $\mathrm{P}<0.05$ was considered to indicate a statistically significant difference.

\section{Results}

Differential expression of NAMPT in normal breast and cancerous tissues. NAMPT protein expression was localized in the cytoplasm and cell membrane of positive tumor or normal cells. Specifically, NAMPT protein was mostly expressed in breast invasive ductal carcinoma as well as in a few adjacent normal mammary glands (Fig. 2). There was no single tumor with completely negative NAMPT expression (Fig. 2). Expression of NAMPT protein was significantly upregulated in breast cancer tissues compared with normal breast tissues $(\mathrm{P}<0.001$; Table I). Then, the association of 

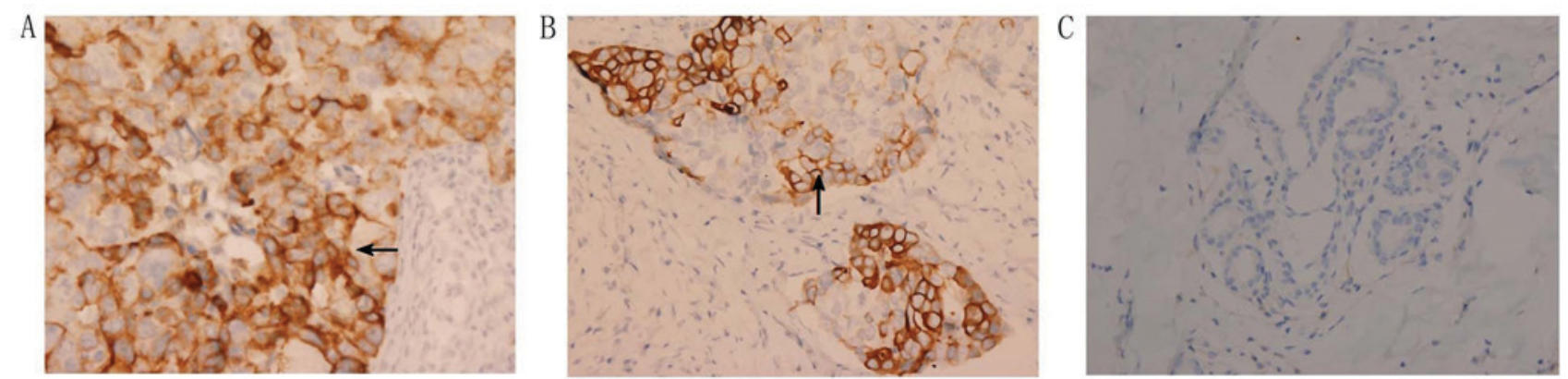

Figure 2. Immunohistochemical analysis of NAMPT expression in normal and cancerous breast tissues. (A) Strong immunostaining of NAMPT protein in breast cancer tissue. Arrow indicates NAMPT positive expression. (B) Strong and moderate immunostaining of NAMPT protein in breast cancer tissue. Arrow indicates NAMPT positive expression. (C) Negative immunostaining of NAMPT in normal breast tissue. Magnification, x400. NAMPT, nicotinamide phosphoribosyltransferase.

Table I. NAMPT expression in breast cancer tissue samples.

\begin{tabular}{lccc}
\hline Group & $\begin{array}{c}\text { High NAMPT } \\
\text { expression }\end{array}$ & $\begin{array}{c}\text { Low or no NAMPT } \\
\text { expression }\end{array}$ & P-value \\
\hline Breast cancer & 40 & 43 & \\
Adjacent & 0 & 83 & 0.001 \\
normal tissues & & & \\
\hline
\end{tabular}

NAMPT, nicotinamide phosphoribosyltransferase.

NAMPT expression with the clinicopathological data from the breast cancer patients was analyzed. The results demonstrated that upregulated NAMPT protein expression was associated with a larger tumor size, lymph node metastasis, advanced clinical TNM stages, and ER and PR expression (Table II).

Association of NAMPT protein expression with survival of breast cancer patients. Next, the association of NAMPT expression with survival of the breast cancer patients was examined. Kaplan-Meier curves, stratified by high vs. low NAMPT expression, revealed that high NAMPT expression was associated with a poor overall and disease-free survival in patients, while breast cancer tissues without or with low NAMPT expression had better disease-free and overall survival rates $(\mathrm{P}=0.010$ and $\mathrm{P}=0.045$; Fig. 3). Furthermore, compared with patients with ER-positive tumors, patients with ER-negative tumors had a significantly worse disease-free survival both in the high NAMPT group and in the low NAMPT group ( $\mathrm{P}=0.022$ and $\mathrm{P}=0.002$, respectively) and overall survival both in the high NAMPT group and in the low NAMPT group ( $\mathrm{P}=0.042$ and $\mathrm{P}=0.019$, respectively) (Figs. 4 and 5).

To evaluate the factors associated with NAMPT expression in these patients, hazard ratios (HRs) were estimated by univariate and multivariate Cox regression analyses (Tables III and IV). For the univariate analysis, the significant factors associated with disease-free survival included tumor stage (HR=52.39, 95\% CI $=11.90-230.65$, $\mathrm{P}<0.001)$, tumor grade $(\mathrm{HR}=2.66,95 \% \mathrm{CI}=1.01-7.01, \mathrm{P}=0.047)$, ER status $(\mathrm{HR}=0.29,95 \% \mathrm{CI}=0.12-0.72, \mathrm{P}=0.007)$ and NAMPT expression $(\mathrm{HR}=3.13,95 \% \mathrm{CI}=1.27-7.73, \mathrm{P}=0.013$; Table III). By contrast, the only significant factor associated with overall
Table II. Association of NAMPT expression with clinicopathological characteristics in breast cancer patients.

\begin{tabular}{|c|c|c|c|}
\hline \multirow[b]{2}{*}{ Variables } & \multicolumn{2}{|c|}{$\begin{array}{l}\text { Level of NAMPT } \\
\text { expression }\end{array}$} & \multirow[b]{2}{*}{ P-value } \\
\hline & Low (n) & High (n) & \\
\hline \multicolumn{4}{|c|}{ Age (years) } \\
\hline$\leq 50$ & 25 & 19 & \\
\hline$>50$ & 18 & 21 & 0.330 \\
\hline \multicolumn{4}{|c|}{ Tumor size $(\mathrm{cm})$} \\
\hline$<2$ & 25 & 12 & \\
\hline$\geq 2$ & 18 & 28 & 0.010 \\
\hline \multicolumn{4}{|c|}{ LN metastasis } \\
\hline No & 36 & 24 & \\
\hline Yes & 7 & 16 & 0.016 \\
\hline \multicolumn{4}{|l|}{ TNM stage } \\
\hline $\mathrm{I} / \mathrm{II}$ & 37 & 26 & \\
\hline III/IV & 6 & 14 & 0.030 \\
\hline \multicolumn{4}{|l|}{ Grade } \\
\hline $\mathrm{I} / \mathrm{II}$ & 34 & 28 & \\
\hline III & 9 & 12 & 0.340 \\
\hline \multicolumn{4}{|c|}{ ER expression } \\
\hline Negative & 6 & 19 & \\
\hline Positive & 37 & 21 & 0.001 \\
\hline \multicolumn{4}{|c|}{ PR expression } \\
\hline Negative & 11 & 23 & \\
\hline Positive & 32 & 17 & 0.003 \\
\hline \multicolumn{4}{|c|}{ HER2 expression } \\
\hline Negative & 24 & 27 & \\
\hline Positive & 19 & 13 & 0.274 \\
\hline
\end{tabular}

NAMPT, nicotinamide phosphoribosyltransferase; LN, lymph node; TNM, tumor-node-metastasis staging; ER, estrogen receptor; PR, progesterone receptor; HER2, human epidermal growth factor receptor 2 .

survival was tumor stage $(\mathrm{HR}=55.02,95 \% \mathrm{CI}=7.12-425.20$, $\mathrm{P}<0.001$; Table IV). However, after adjusting for the patient 
A

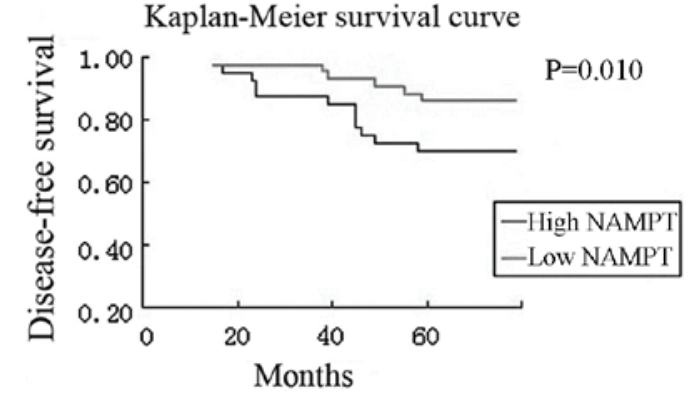

B

Kaplan-Meier survival curve

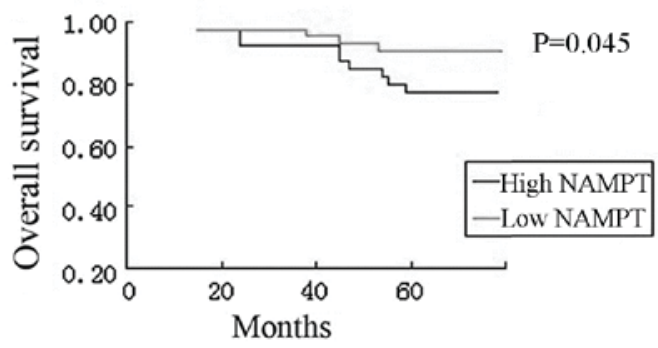

Figure 3. Kaplan-Meier survival curves stratified by NAMPT expression in breast cancer patients. (A) Disease-free survival. (B) Overall survival. NAMPT, nicotinamide phosphoribosyltransferase.

A

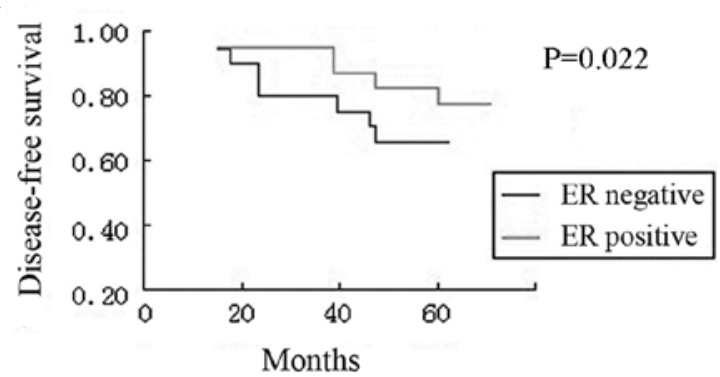

B

Kaplan-Meier survival curve (high NAMPT)

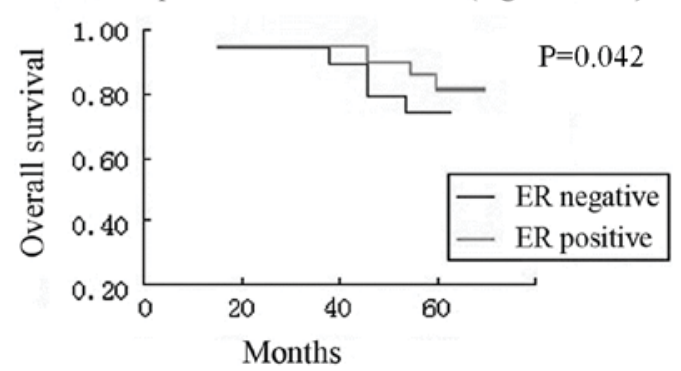

Figure 4. Kaplan-Meier curves stratified by ER expression in patients with high tumor expression of NAMPT. (A) Disease-free survival. (B) Overall survival. ER, estrogen receptor; NAMPT, nicotinamide phosphoribosyltransferase.

age at diagnosis, tumor stage, tumor grade, ER status, HER2 status, and NAMPT expression by multivariate Cox regression analysis, only tumor stage (HR=63.42, 95\% CI=12.91-311.48, $\mathrm{P}<0.001$ and $\mathrm{HR}=115.26,95 \% \mathrm{CI}=13.24-1003.63$, $\mathrm{P}<0.001$, respectively) and NAMPT expression ( $\mathrm{HR}=0.53$, 95\% $\mathrm{CI}=0.17-1.59, \mathrm{P}=0.255$ and $\mathrm{HR}=0.20,95 \% \mathrm{CI}=0.04-0.89$, $\mathrm{P}=0.034$, respectively) were significant independent predictors of disease-free and overall survival in the breast cancer patients (Tables III and IV).
A Kaplan-Meier survival curve (low NAMPT)

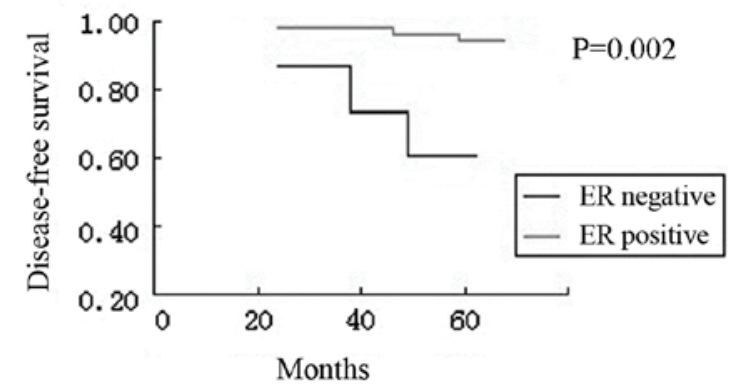

B

Kaplan-Meier survival curve (low NAMPT)

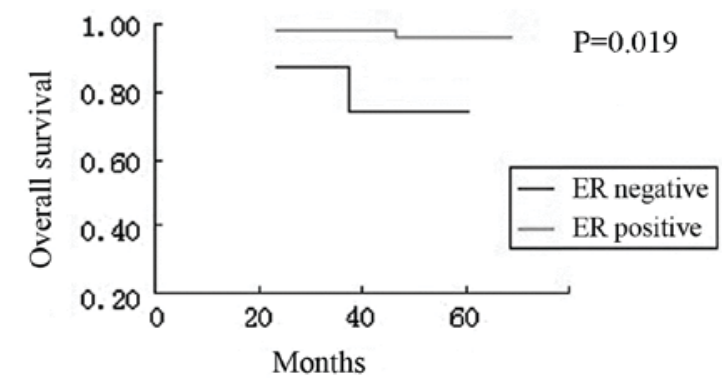

Figure 5. Kaplan-Meier curves stratified by ER expression in patients with low tumor expression of NAMPT. (A) Disease-free survival. (B) Overall survival. ER, estrogen receptor; NAMPT, nicotinamide phosphoribosyltransferase.

\section{Discussion}

Although early detection and treatment of breast cancer have significantly advanced compared with decades ago, more research is needed for the molecular diagnosis and prognosis prediction of breast cancer. In the current study, NAMPT expression was analyzed and associated with breast cancer development and progression in order to potentially identify it as a novel biomarker for breast cancer. The present data demonstrated that NAMPT expression was significantly higher in breast cancer tissues compared with normal mammary gland tissues, and that upregulated NAMPT protein expression was associated with a larger tumor size, advanced clinical TNM stages, lymph node metastasis, and ER and PR expression. Furthermore, NAMPT expression was associated with poor overall and disease-free survival in patients, whereas breast cancer without or with low NAMPT expression had a better disease-free and overall survival. In conclusion, the present findings suggest that NAMPT upregulation may contribute to breast cancer development and progression and that detection of NAMPT protein levels may serve as a biomarker for the early detection and prognosis prediction of breast cancer.

Indeed, NAMPT has been reported to be overexpressed in several types of human cancer and to induce resistance to therapy (3), suggesting that NAMPT could be used as a chemotherapeutic target (3). Translationally, NAMPT might be a useful biomarker for carcinogenesis and tumor progression (4). For example, upregulated NAMPT expression has been associated with an increase in melanoma volume, early metastases, and tumor cell de-differentiation (11). In addition, NAMPT overexpression has been observed in hematological malignancies, such as lymphomas, and is associated with aggressive phenotypes of malignant lymphoma (12). In solid 
Table III. Univariate and multivariate analyses of disease-free survival in breast cancer cases.

\begin{tabular}{|c|c|c|c|c|c|c|}
\hline \multirow[b]{2}{*}{ Variables } & \multicolumn{3}{|c|}{ Univariate } & \multicolumn{3}{|c|}{ Multivariate } \\
\hline & HR & $95 \% \mathrm{CI}$ & P-value & HR & $95 \% \mathrm{CI}$ & P-value \\
\hline \multicolumn{7}{|c|}{ Age (years) } \\
\hline$>50$ & 0.80 & $0.32-1.98$ & 0.622 & 0.91 & $0.32-2.59$ & 0.861 \\
\hline$\leq 50$ & 1.00 & & & 1.00 & & \\
\hline \multicolumn{7}{|l|}{ Stage } \\
\hline III/IV & 52.39 & $11.90-230.65$ & $<0.001$ & 63.42 & $12.91-311.48$ & $<0.001$ \\
\hline $\mathrm{I} / \mathrm{II}$ & 1.00 & & & 1.00 & & \\
\hline \multicolumn{7}{|l|}{ Grade } \\
\hline III/IV & 2.66 & $1.01-7.01$ & 0.047 & 0.98 & $0.33-2.95$ & 0.967 \\
\hline $\mathrm{I} / \mathrm{II}$ & 1.00 & & & 1.00 & & \\
\hline \multicolumn{7}{|l|}{ ER } \\
\hline Positive & 0.29 & $0.12-0.72$ & 0.007 & 0.49 & $0.16-1.54$ & 0.225 \\
\hline Negative & 1.00 & & & 1.00 & & \\
\hline \multicolumn{7}{|l|}{ HER 2} \\
\hline Positive & 0.97 & $0.38-2.45$ & 0.942 & 0.81 & $0.28-2.32$ & 0.687 \\
\hline Negative & 1.00 & & & 1.00 & & \\
\hline \multicolumn{7}{|l|}{ NAMPT } \\
\hline High & 3.13 & $1.27-7.73$ & 0.013 & 0.53 & $0.17-1.59$ & 0.255 \\
\hline Low & 1.00 & & & 1.00 & & \\
\hline
\end{tabular}

HR, hazard ratio; CI, confidence interval; ER, estrogen receptor; HER2, human epidermal growth factor receptor 2; NAMPT, nicotinamide phosphoribosyltransferase.

Table IV. Univariate and multivariate analyses of overall survival in breast cancer cases.

\begin{tabular}{|c|c|c|c|c|c|c|}
\hline \multirow[b]{2}{*}{ Variables } & \multicolumn{3}{|c|}{ Univariate } & \multicolumn{3}{|c|}{ Multivariate } \\
\hline & HR & $95 \%$ CI & P-value & HR & $95 \% \mathrm{CI}$ & P-value \\
\hline \multicolumn{7}{|c|}{ Age (years) } \\
\hline$>50$ & 0.96 & $0.32-2.86$ & 0.941 & 0.82 & $0.24-2.81$ & 0.754 \\
\hline$\leq 50$ & 1.00 & & & 1.00 & & \\
\hline \multicolumn{7}{|l|}{ Stage } \\
\hline III/IV & 55.02 & $7.12-425.20$ & $<0.001$ & 115.26 & $13.24-1003.63$ & $<0.001$ \\
\hline $\mathrm{I} / \mathrm{II}$ & 1.00 & & & 1.00 & & \\
\hline \multicolumn{7}{|l|}{ Grade } \\
\hline III/IV & 2.56 & $0.79-8.31$ & 0.118 & 0.90 & $0.24-3.33$ & 0.872 \\
\hline $\mathrm{I} / \mathrm{II}$ & 1.00 & & & 1.00 & & \\
\hline \multicolumn{7}{|l|}{ ER } \\
\hline Positive & 0.59 & $0.19-1.81$ & 0.359 & 0.80 & $0.19-3.39$ & 0.766 \\
\hline Negative & 1.00 & & & 1.00 & & \\
\hline \multicolumn{7}{|l|}{ HER2 } \\
\hline Positive & 0.72 & $0.22-2.35$ & 0.591 & 0.80 & $0.22-2.91$ & 0.731 \\
\hline Negative & 1.00 & & & 1.00 & & \\
\hline \multicolumn{7}{|l|}{ NAMPT } \\
\hline High & 1.34 & $0.41-4.35$ & 0.628 & 0.20 & 0.04-0.89 & 0.034 \\
\hline Low & 1.00 & & & 1.00 & & \\
\hline
\end{tabular}

HR, hazard ratio; CI, confidence interval; ER, estrogen receptor; HER2, human epidermal growth factor receptor 2; NAMPT, nicotinamide phosphoribosyltransferase. 
tumors, NAMPT is upregulated in prostate, gastric, and colorectal cancers (13-15), and NAMPT inhibition reduces the growth and invasiveness of prostate cancer cells in vitro (15). A previous study has reported that detection of NAMPT, vascular endothelial growth factor, and HER2 could be useful as a biomarker panel for the diagnosis and prognosis of human breast cancer (16). The current study further confirmed the overexpression of NAMPT protein in invasive breast cancer and it association with breast cancer progression and prognosis. Of note, a previous in vitro study has demonstrated that NAMPT expression influences breast cancer cell metastatic activity and adhesion by inhibition of integrin function (17).

Breast cancer exhibits many molecular alterations; for example, $B R C A$ mutations are the main hereditary factor in the development of breast cancer, while the expression of poly (ADP-ribose) polymerase 1 (PARP1) contributes to the BRCA1 phenotype in basal-like and triple-negative breast cancers (18). In addition, BRCA1-mediated NAD synthesis is largely responsible for PARP1 activity in breast cancer cells, while NAMPT regulates NAD levels towards aberrant PARP1 activity (19). Indeed, Bajrami et al (20) have reported that an NAMPT inhibitor in combination with a PARP inhibitor has a significant therapeutic potency in nude mouse triple-negative breast cancer cell xenografts. NAMPT was originally cloned as a putative cytokine to enhance the maturation of B lymphocyte precursors (21). Later, it was reported that NAMPT is able to promote both B lymphocyte and vascular smooth muscle cell maturation as well as inhibit neutrophil apoptosis (22). A previous study has demonstrated that NAMPT expression can predict recurrence-free survival of lung and breast cancer patients (23). Another recent study has revealed a novel mechanism by which breast cancer cells are able to protect themselves from glucose deprivation-induced oxidative stress through NAMPT to maintain NADPH levels (24). Together, these findings indicate that NAMPT overexpression can alter human immune responses and promote breast cancer progression.

FK866 (also known as APO866) is a small-molecule inhibitor of NAMPT with potency and selectivity; in vitro, it is able to suppress the growth of cancer cells and induce tumor cells to undergo apoptosis through NAD depletion (25). Notably, FK866 can selectively inhibit various types of cancer cells, but not normal cells (25). The current data indicate that breast cancer may be another organ site where NAMPT inhibitors may be useful in the clinic.

To fully understand the physiological relevance of NAMPT expression in normal and cancerous mammary gland cells, further research will be necessary. For example, future studies may include information on subtypes of breast cancer and expression of other molecules to examine the associations with NAMPT expression. In addition, the data from the current study need to be verified using a larger sample size before NAMPT is used clinically as a biomarker. Further studies will also assess NAMPT in mediating breast cancer progression and the potential of targeting NAMPT as a therapeutic strategy in breast cancer.

\section{Acknowledgements}

The authors would like to thank the staff of the Genetic Disease Research Institution, Xi'an Jiaotong University (Xi'an, China) for their technical assistance.

\section{Funding}

This study was supported in part by a grant from Weihai Municipal Hospital Research Fund (grant no. WHSLYYZBB2016-107).

\section{Availability of data and materials}

The analyzed datasets generated during the study are available from the corresponding author upon reasonable request.

\section{Author's contributions}

SJZ and TQB generated and analyzed data for all figures; CXQ generated and analyzed data for Tables I and II; XQY generated data for Tables III and IV; KP verified statistical analysis; TQB wrote the manuscript, which was approved by all authors; SJZ supervised the project and secured funding.

\section{Ethics approval and consent to participate}

This study was approved by the Ethics Committee of Weihai Municipal Hospital (Weihai, China), and informed consent was obtained from all patients.

\section{Consent for publication}

Not applicable.

\section{Competing interests}

The authors declare that they have competing interests.

\section{References}

1. Siegel RL, Miller KD and Jemal A: Cancer statistics, 2016. CA Cancer J Clin 66: 7-30, 2016.

2. Chen W, Zheng R, Baade PD, Zhang S, Zeng H, Bray F, Jemal A, Yu XQ and He J: Cancer statistics in China, 2015. CA Cancer J Clin 66: 115-132, 2016.

3. Shackelford RE, Mayhall K, Maxwell NM, Kandil E and Coppola D: Nicotinamide phosphoribosyltransferase in malignancy: A review. Genes Cancer 4: 447-456, 2013.

4. Bi TQ and Che XM: Nampt/PBEF/visfatin and cancer. Cancer Biol Ther 10: 119-125, 2010.

5. Chini CC, Guerrico AM, Nin V, Camacho-Pereira J, Escande C, Barbosa MT and Chini EN: Targeting of NAD metabolism in pancreatic cancer cells: Potential novel therapy for pancreatic tumors. Clin Cancer Res 20: 120-130, 2014.

6. Folgueira MA, Carraro DM, Brentani H, Patrão DF, Barbosa EM, Netto MM, Caldeira JR, Katayama ML, Soares FA, Oliveira CT, et al: Gene expression profile associated with response to doxorubicin-based therapy in breast cancer. Clin Cancer Res 11: 7434-7443, 2005.

7. Bi TQ, Che XM, Liao XH, Zhang DJ, Long HL, Li HJ and Zhao W: Overexpression of Nampt in gastric cancer and chemopotentiating effects of the Nampt inhibitor FK866 in combination with fluorouracil. Oncol Rep 26: 1251-1257, 2011.

8. Long HL, Che XM, BI TQ, Li HJ, Liu JS and Li DW: The expression of nicotinamide phosphoribosyl transferase and vascular endothelial growth factor-A in gastric carcinoma and their clinical significance. Zhonghua Wai Ke Za Zhi 50: 839-842, 2012

9. Tavassoli FA: Pathology of the Breast. McGraw-Hill Medical, New York, NY, USA, pp254-397, 1999.

10. Edge SB, Byrd DR, Compton CC, Fritz AG, Greene FL and Trotti A (eds). AJCC Cancer Staging Manual. 7th ed. New York, Springer, 2010. 
11. Maldi E, Travelli C, Caldarelli A, Agazzone N, Cintura S, Galli U, Scatolini M, Ostano P, Miglino B, Chiorino G, et al: Nicotinamide phosphoribosyltransferase (NAMPT) is over-expressed in melanoma lesions. Pigment Cell Melanoma Res 26: 144-146, 2013.

12. Olesen UH, Hastrup N and Sehested M: Expression patterns of nicotinamide phosphoribosyltransferase and nicotinic acid phosphoribosyltransferase in human malignant lymphomas. APMIS 119: 296-303, 2011.

13. Nakajima TE, Yamada Y, Hamano T, Furuta K, Gotoda T, Katai H, Kato K, Hamaguchi T and Shimada Y: Adipocytokine levels in gastric cancer patients: Resistin and visfatin as biomarkers of gastric cancer. J Gastroenterol 44: 685-690, 2009.

14. Nakajima TE, Yamada Y, Hamano T, Furuta K, Matsuda T, Fujita S, Kato K, Hamaguchi T and Shimada Y: Adipocytokines as new promising markers of colorectal tumors: Adiponectin for colorectal adenoma, and resistin and visfatin for colorectal cancer. Cancer Sci 101: 1286-1291, 2010.

15. Wang B, Hasan MK, Alvarado E, Yuan H, Wu H and Chen WY: NAMPT overexpression in prostate cancer and its contribution to tumor cell survival and stress response. Oncogene 30: 907-921, 2011.

16. Zhu Y, Guo M, Zhang L, Xu T, Wang L and Xu G: Biomarker triplet NAMPT/VEGF/HER2 as a de novo detection panel for the diagnosis and prognosis of human breast cancer. Oncol Rep 35: 454-462, 2016.

17. Santidrian AF, LeBoeuf SE, Wold ED, Ritland M, Forsyth JS and Felding BH: Nicotinamide phosphoribosyltransferase can affect metastatic activity and cell adhesive functions by regulating integrins in breast cancer. DNA Repair 23: 79-87, 2014

18. Domagala P, Huzarski T, Lubinski J, Gugala K and Domagala W: PARP-1 expression in breast cancer including $B R C A 1$-associated, triple negative and basal-like tumors: Possible implications for PARP-1 inhibitor therapy. Breast Cancer Res Treat 127: 861-869, 2011.
19. Li D, Bi FF, Chen NN, Cao JM, Sun WP, Zhou YM, Li CY and Yang Q: A novel crosstalk between BRCA1 and poly (ADP-ribose) polymerase 1 in breast cancer. Cell Cycle 13: 3442-3449, 2014

20. Bajrami I, Kigozi A, van Weverwijk A, Brough R, Frankum J, Lord CJ and Ashworth A: Synthetic lethality of PARP and NAMPT inhibition in triple-negative breast cancer cells. EMBO Mol Med 4: 1087-1096, 2012.

21. Samal B, Sun Y, Stearns G, Xie C, Suggs S and McNiece I: Cloning and characterization of the cDNA encoding a novel human pre-B-cell colony-enhancing factor. Mol Cell Biol 14: 1431-1437, 1994.

22. Jia SH, Li Y, Parodo J, Kapus A, Fan L, Rotstein OD and Marshall JC: Pre-B cell colony-enhancing factor inhibits neutrophil apoptosis in experimental inflammation and clinical sepsis. J Clin Invest 113: 1318-1327, 2004.

23. Zhou T, Wang T and Garcia JG: Expression of nicotinamide phosphoribosyltransferase-influenced genes predicts recurrence-free survival in lung and breast cancers. Sci Rep 4: 6107, 2014.

24. Hong SM, Park CW, Kim SW, Nam YJ, Yu JH, Shin JH, Yun CH, Im SH, Kim KT, Sung YC and Choi KY: NAMPT suppresses glucose deprivation-induced oxidative stress by increasing NADPH levels in breast cancer. Oncogene 35: 3544-3554, 2016.

25. Zerp SF, Vens C, Floot B, Verheij M and van Triest B: NAD ${ }^{+}$ depletion by APO866 in combination with radiation in a prostate cancer model, results from an in vitro and in vivo study. Radiother Oncol 110: 348-354, 2014. 